\title{
Cyclic Cushing's Disease: Pitfalls in the Diagnosis and Problems with the Pathogenesis
}

The diagnosis of typical Cushing's syndrome is not very difficult. However, in unusual patients with rhythmic cortisol production between high and normal levels, the diagnosis is not straightforward. Such disorders have been termed "cyclic" or "intermittent" Cushing's syndrome. In the former, the length of cycles varies from patient to patient but it is remarkably constant for each individual patient with a cycle length of 1-86 days. It is diagnosed by showing 3 peaks and 2 troughs of cortisol production. In the latter, the cycle of cortisol production is irregular, ranging from 10 episodes of relapse in 3 months to 2 episodes of exerbation in 5 years. In some cases, however, it is not feasible to determine the precise periodicity because of its prolonged nature. Hence, both disorders could be unified to an entity as Cushing's syndrome with periodic hormonogenesis. In this issue of the Journal, a typical case of cyclic Cushing's disease was reported by Adachi et al (1).

See also p 207.

Of interest is that a low dose of bromocriptine was effective in that case. In Cushing's disease with periodic hormonogenesis, the presence of pitfalls in the diagnosis has been noted, and also pathogenesis of periodic hormone production has been emphasized.

When the trough of cortisol production persists for a long time, these patients show complete remission clinically and biochemically, and the standard diagnostic tests for Cushing's syndrome such as dexamethasone suppression test, metyrapone test, diurnal variation of plasma cortisol and corticotropinreleasing hormone $(\mathrm{CRH})$ test become normal. These patients often present edema, proxymal myopathy and hypokalemia rather than typical cushingoid appearance in the recurrent phase after a long-term remission (2). These laboratory and clinical features are misleading for the diagnosis. Multiple determinations of plasma levels and/or urinary excretion of hormones and long-term observation alleviates this pitfall.

Concerning the pathogenesis of Cushing's disease, the controversy that the primary etiology of corticotroph adenomas lies within the pituitary gland or the hypothalamus has been debated. Although recent studies using modern molecular biology strongly support the concept that a somatic mutation is a requisite event in adenoma formation (3), clinical and laboratory observations in cyclic Cushing's disease raise very interesting questions on the pathogenesis, and the possibility of abnormal hypothalamic control in the disease is suggested.
That is, one of the facts that support the hypothalamic dysfunction theory is a high recurrence rate following successful transsphenoidal adenomectomy. In the case of Adachi et al (1), hypercortisolism recurred following the complete remission for 7 months with Hardy's operation. A review of the literature on patients with cyclic Cushing's disease showed that recurrence rate by Hardy's operation was as high as $63 \%$ (10 out of 16 patients), and remission was only $25 \%$ (4 out of 16 cases) (2). In the patients with recurrent hypercortisolism after transsphenoidal adenomectomy, the possibility of cyclic Cushing's disease should be considered. Furthermore, neuropharmacological agents including bromocriptine, cyproheptadine and sodium valproate, which are believed to act via alterations in neural pathways regulating various aspects of CRH release, are effective in some cases (2). These findings also suggest hypothalamic dysregulation. On bromocriptine, it was administered at very large doses, $40 \mathrm{mg} / \mathrm{day}$, in the effective case (4). The report by Adachi et al (1) is the first report of a patient successfully treated with a low dose of bromocriptine. In addition to circadian rhythm, an ultradian (3-8 hour cycle) and infradian (20 to 30 days) pattern of adrenocorticotropic hormone (ACTH) secretion has long been recognized in humans. It seems likely that such cyclicity of ACTH release is under central nervous system control. Thus, periodic hormonogenesis might suggest the hypothalamic origin as the pathogenesis of such Cushing's disease.

On the other hand, some reports suggest that neuropharmacological agents act directly on the pituitary. Although the most common cause of cyclic or intermittent Cushing's syndrome is pituitary adenoma and the rate is above $80 \%$ in about 60 reported cases (2), ectopic ACTH syndrome and Cushing's syndrome due to adrenal adenoma are also included. And hence, the findings mentioned in the above paragraph are not always compelling evidence of hypothalamic dysfunction in cyclic Cushing's disease. Recently, several cases of ectopic CRH-producing tumor associated with hypercortisolism have been reported. In those cases, the pathological findings of the pituitary were hyperplasia but not pituitary adenoma (5). This finding does not support the hypothesis for the etiology of cyclic Cushing's disease that centrally induced chronic hypersecretion of CRH may lead to microadenoma formation, because pituitary hyperplasia was found in few patients with cyclic Cushing's disease (2). Furthermore, the examination of clonality of ACTH-secreting pituitary microadenoma from usual Cushing's disease was confirmed to be monoclonal, indicating that 
hypersecretion of CRH is not involved in pituitary microadenoma formation (3). On the contrary, in transgenic mice model with overexpression of $\mathrm{CRH}$, pituitary histology showed corticotroph microadenoma in one report (6), suggesting an important role of chronic $\mathrm{CRH}$ production for the pituitary microadenoma formation. Determination of the clonality of the pituitary micro-adenoma obtained from cyclic Cushing's disease will play a key role in the elucidation of the pathogenesis of Cushing's disease associated with periodic hormonogenesis in the future.

Keigo YASUDA, MD Third Department of Internal Medicine, Gifu University School of Medicine, 40 Tsukasa-machi, Gifu 500

\section{References}

1) Adachi M, Takayanagi R, Yanase T, et al. Cyclic Cushing's disease in long-term remission with a daily low dose of bromocriptine. Intern Med 35: 207, 1996.

2) Yasuda K, Miura K. Unique type of Cushing's disease in clinical profile: Cyclic Cushing's syndrome and Cushing's disease with favorable outcome to a high daily dose of bromocriptine. Folia Endocrinol 70: 11, 1994 (in Japanese).

3) Biller BMK, Alexander JM, Zervas NT, Hedley-White ET, Arnold A, Klibanski A. Clonal origins of adrenocorticotropin-secreting pituitary tissue in Cushing's disease. J Clin Endocrinol Metab 75: 1303, 1992.

4) Mercado-Asis LB, Murayama M, Yamakita N, et al. Cortisol-suppressible dexamethasone-non-suppressible cyclic Cushing's disease with evidence of clinical and biochemical remission with bromocriptine. Endocrinol Jpn 38: 315, 1991.

5) Wajchenberg BL, Mendonca BB, Liberman B, et al. Ectopic adrenocorticotropic hormone syndrome. Endocr Rev 15: 752, 1994.

6) Helseth A, Siegal GP, Haug E, Bautch VL. Transgenic mice that develop pituitary tumors. A model for Cushing's disease. Am J Path 140: 1071, 1992. 